\title{
Abhandlungen
}

\author{
Benjamin Friedländer
}

\section{Öffentliche Unternehmen und Beteiligungsmanagement im kommunalpolitischen Prozess: Konzeptionelle Fundierung und Befunde einer Befragung und Dokumentenanalyse}

Öffentliche Unternehmen; Beteiligungsmanagement; Policy; kommunalpolitischer Prozess; Polity; Politics; Governancemechanismen und Koordination

Mit Blick auf die wissenschaftliche Literatur ist festzustellen, dass sich die Diskussion um öffentliche Unternehmen vornehmlich auf isolierte Aspekte der betriebswirtschaftlichen Steuerung und Kontrolle sowie auf institutionenökonomische Analysen konzentriert. Dem Untersuchungsgegenstand fehlt es merklich an einer Einordnung in politikwissenschaftliche Kategorien und politische Rahmenbedingungen, was thematisch insb. den Beitrag öffentlicher Unternehmen und der mit ihrer Steuerung und Kontrolle befassten Akteure in kommunalpolitischen Prozessen betrifft. Auf Grundlage eines Analyse- und Interpretationsrahmens aus Policy-, Politicsund Polity-Perspektive sowie explorativen Befunden aus einer mündlichen Befragung kommunaler Entscheidungsträger und einer Dokumentenanalyse wird daher $u$. a. der Frage nachgegangen, welche Rolle öffentliche Unternehmen in einzelnen Phasen des politischen Prozesses leisten, wie diese Rolle und die damit einhergehenden Interaktionsmuster zu bewerten sind und welchen Einfluss das Beteiligungsmanagement bei Entscheidungsprozessen, Konfliktregelung und Konsensbildung hat.

\section{Einleitung}

Die Wahrnehmung öffentlicher Aufgaben ist in den letzten Jahrzehnten auf immer mehr Institutionen außerhalb der öffentlichen Kernverwaltung verlagert worden. Dies hat zur Folge, dass sich die Institutionenlandschaft deutlich ausdifferenziert und in den meisten Kommunen zu einem breiten Portfolio an Beteiligungen mit zum Teil komplexen konzernähnlichen Strukturen geführt hat (Röber 2012, S. 15ff.; Grossi/Reichard 2008, S. 598ff.). Bereits Anfang der 1990er Jahre ist durch die Kommunale Gemeinschaftsstelle für Verwaltungsmanagement (KGSt) darauf hingewiesen worden, dass der Anteil kommunaler Beteiligungen in einigen Großstädten an der Zahl der Beschäftigten sowie dem Volumen an Ausgaben und Investitionen bei etwa 50 
Prozent liegt (KGSt 1993, S. 11). Aktuellere Studien unterstreichen diese Entwicklung bis heute, ebenfalls für den Bereich der kommunalen Schulden (u. a. Hesse/Lenk/Starke 2017, S. 23). Insoweit ist die Relevanz öffentlicher Unternehmen für die Aufgabenerfüllung mittlerweile unbestritten und für die kommunale Ebene hinreichend gut belegt.

Mit Blick auf die wissenschaftliche Literatur stellt man allerdings fest, dass sich Diskussion und Analyse öffentlicher Unternehmen in den letzten Jahren vornehmlich auf isolierte Aspekte der betriebswirtschaftlichen Steuerung und Kontrolle sowie auf institutionenökonomische Kategorien konzentriert. Bis auf sehr wenige Ausnahmen (z. B. Röber 2008, Schröter/Röber 2017 sowie der Beitrag von Schröter/Röber/Röber in diesem Heft) mangelt es dem Untersuchungsgegenstand merklich an der Einordnung in politikwissenschaftliche bzw. politische Rahmenbedingungen, z. B. in Hinblick auf die politische Steuerung sowie Steuerbarkeit und Steuerungsfähigkeit solcher Organisationen (zu den Begriffen Naschold u. a. 2000).

Besonders auffällig für die kommunale Ebene ist, dass Fragen nach dem Beitrag öffentlicher Unternehmen und der mit ihrer Steuerung und Kontrolle befassten Akteure (Beteiligungsmanagement) im politischen Entscheidungsprozess sowie den mit diesen Akteuren einhergehenden Interaktionsformen und Einflussmöglichkeiten bislang wenig Aufmerksamkeit gewidmet wurde. Diese Lücke ist nicht nur für die vornehmlich instrumentell ausgerichtete Public-Management-Forschung zu konstatieren, sondern mindestens genauso für Bereiche der Governanceund Policy-Forschung. Was das Feld der Governance-Forschung anbetrifft, so konzentriert man sich bei Fragen nach der organisationspolitischen Ausgestaltung öffentlicher Leistungserbringung trotz der oben skizzierten institutionellen Entwicklungen weitestgehend auf Akteure aus Politik, Verwaltung, sog. Dritte und Zivilgesellschaft. Dass es zwischen diesen Institutionen und Akteuren aber eine Vielzahl an verselbständigten und ausgelagerten Aufgabenträgern gibt, bleibt vielfach unberücksichtigt. Ähnlich sieht es für die Policy-Forschung aus. Auch hier konzentriert man sich im Rahmen von Analysen der am Policy Making beteiligten Akteure und deren Interaktion vornehmlich auf die Sphären von Politik und Kernverwaltung, kaum aber auf die Integration und den Einfluss von verselbständigten und ausgelagerten Organisationseinheiten (für die Bundesebene am ehesten noch Bach 2012, S. 211ff.). Diese Feststellung unterstreicht nicht nur den Bedarf nach einer stärker politikwissenschaftlichen und vor allem policybezogenen Betrachtung öffentlicher Unternehmen, sondern offenbart auch viele ,weiße Flecken“ in einer mitunter zu isoliert und wenig interdisziplinär ausgerichteten Analyse des öffentlichen Sektors im Allgemeinen (hierzu Friedländer 2019).

Vor diesem Hintergrund wird im folgenden Abschnitt II die Bedeutung der unterschiedlichen Dimensionen des Politikbegriffs sowie des Policy Cycle als wichtiger Analyse- und Interpretationsrahmen für die Betrachtung und Bewertung des Beitrags öffentlicher Unternehmen im kommunalpolitischen Prozess herausgearbeitet. Im Anschluss (Abschnitt III) werden auf Grundlage von explorativen Befunden aus einer mündlichen Befragung kommunaler Entscheidungsträger sowie einer Dokumentenanalyse mögliche Antworten auf die Frage gegeben, welchen Beitrag öffentliche Unternehmen in den Phasen der Problemwahrnehmung und des Agenda Setting sowie der Politikformulierung leisten und wie diese Rolle zu bewerten ist. Abschnitt IV widmet sich den Einflussmöglichkeiten eines Beteiligungsmanagements im Rahmen von Entscheidungsprozessen, Konfliktregelung und Konsensbildung im kommunalpolitischen Pro- 
zess und zeigt auf, welche unterschiedlichen Governanceprinzipien bzw. Koordinationsmechanismen hierbei zum Einsatz kommen können.

Den folgenden Ausführungen liegen im Wesentlichen Ergebnisse einer Untersuchung und Bewertung der kommunalen Gesamtsteuerung öffentlicher Aufgaben aus Friedländer 2019 zugrunde, im Rahmen derer u. a. der Frage nachgegangen wurde, welche Rolle öffentliche Unternehmen und die mit ihrer Steuerung und Kontrolle befassten Akteure in kommunalpolitischen Entscheidungsprozessen spielen. Die empirische Untersuchung basiert auf einer leitfadengestützten Befragung von 25 Personen aus Kommunalpolitik, Kommunalverwaltung, kommunalen Unternehmen und Nonprofit-Organisationen sowie auf einer Analyse öffentlich zugänglicher Strategie- und Koordinationsdokumente (zur genaueren Erläuterung des methodischen Vorgehens siehe Friedländer 2019, S. 206ff.).

\section{Policy-, Politics- und Polity-Perspektive als Analyse- und Interpretationsrahmen}

Die Notwendigkeit eines policy-orientierten Analyse- und Interpretationsrahmens wird deutlich, wenn man genauer darauf schaut, was der Terminus „Policy“ im Einzelnen beinhaltet. Im Allgemeinen ist festzuhalten, dass Policy die inhaltliche Dimension von Politik beschreibt und dabei bewusst sowohl Entscheidungen als auch Handlungen zur Realisierung kommunalpolitischer Ziele einschließt (Jann 1981, S. 6 f.; Heclo 1972, S. 84). Mit anderen Worten ermöglicht eine Policy-Analyse, die Rolle der Kommunalwirtschaft nicht ausschließlich auf die Stufe der öffentlichen Leistungserbringung zu reduzieren, sondern Untersuchungsperspektiven auf die vorgelagerten kommunalpolitischen Entscheidungsstufen auszudehnen.

Betrachtet man öffentliche Unternehmen aus Policy-Perspektive geht es daher nicht nur um das „Was“ und „Wie“ ihrer öffentlichen Aktivitäten, sondern auch um das „Warum“ und „Wozu“, wobei Determinanten und Einflussfaktoren sowie Folgen und Wirkungen ihres öffentlichen Zwecks in den Fokus rücken. „Policy“ meint in diesem Zusammenhang daher auch die Art und Weise der kommunalen Problemverarbeitung durch öffentliche Unternehmen. Insoweit liegt das Hauptinteresse einer Policy-Perspektive nicht nur auf der inhaltlichen Dimension von Policies sondern ebenso auf dem Zusammenhang zwischen prozessualen und strukturellen Bedingungen von Politikformulierung und -durchführung, um dadurch den Entscheidungsbeitrag öffentlicher Unternehmen im kommunalpolitischen Prozess umfassend herausarbeiten zu können (grundlegend Jann 1981, S. 6ff.; Scharpf 1973 b, S. 16).

Im Rahmen des sog. Policy Cycle ${ }^{1}$ wird kommunales Policy Making in die Prozessstufen Politikwahrnehmung und Agenda Setting, Politikformulierung und Entscheidung, Politikimplementierung, Politikevaluierung und ggf. Politikterminierung unterteilt. Der Policy Cycle wird unter idealtypischer Annahme als ein kaum abgeschlossener Prozess der Problembearbeitung und -verarbeitung verstanden, welcher, auch wenn es kurzfristige Terminierungen geben kann, immer wieder bestehende Policies modifiziert, ergänzt, mit diesen interagiert oder konkurriert.

1 In der Literatur werden verschiedene Phasenmodelle des Policy Making herangezogen und kontrovers diskutiert (zur Übersicht und Diskussion der Ansätze z. B. Jann 1981, S. 30). 
Eine Orientierung am Policy Cycle, so wird in der Literatur konstatiert, trägt maßgeblich dazu bei, komplexe Phänomene hinsichtlich ihrer Voraussetzungen, Einflussfaktoren und Ergebnisse differenziert zu untersuchen. Gerade für Aussagen über interne Dynamiken sowie spezifische Eigenarten und Ursachen von kommunalen Policy-Prozessen bietet der Policy Cycle eine unerlässliche Analyseperspektive (Mayntz 1983, S. 7ff.; Jann/Wegrich 2007, S. 43ff.).

Phasengestützte Policy-Prozesse eröffnen die Möglichkeit, strukturiert auf Akteure und Akteurskonstellationen zu blicken. Kommunalpolitische Prozesse sind als Ergebnis der Interaktion von individuellen und korporativen Akteuren zu verstehen, die bestimmte Fähigkeiten besitzen, durch bestimmte kognitive und normative Orientierungen geprägt sind und deren Handeln in einem bestimmten institutionellen Kontext und unter gegebenen Rahmenbedingungen stattfindet (Scharpf 2000, S. 75). Bezieht man dieses Verständnis exemplarisch auf den für die Kommunalwirtschaft vergleichsweise bedeutsamen Bereich der Energieversorgung, stellt sich z. B. die Frage, wie eine Kommune es bei der recht komplexen Akteursstruktur und Zielsystematik in diesem Politikfeld (u. a. Energiewende, dezentrale Versorgungsstrukturen, $\mathrm{CO}_{2}$-Reduzierung, Energieeffizienz) schaffen kann, unterschiedliche Ansprüche, Interessen und Vorgaben von übergeordneten föderalen Ebenen, politischen Fraktionen, verschiedenen Verwaltungsbereichen (u. a. Umwelt, Stadtentwicklung, Wirtschaft und Finanzen, ggf. Agenda 21-Büro), kommunalen Energieversorgungs-, Wohnungsbau- und Nahverkehrsunternehmen, aber auch von umwelt- und naturschutzbezogenen Nonprofit-Organisationen zu bündeln und auszubalancieren. Mit dem Policy Cycle kann bei solchen Fragestellungen ein Untersuchungsschwerpunkt bewusst darauf gelegt werden, dass kommunales Policy Making von einer Vielzahl an Akteuren sowie Steuerungs- und Koordinationsinstrumenten und damit von verschiedenen Facetten der Politics, Polity, Ökonomie bzw. Ökonomisierung, Legalität und Gesetzesmäßigkeit überlagert wird bzw. mit diesen Faktoren in Wechselbeziehungen steht (Jann 1984, S. 37 f.).

Die Grundidee dieses Ansatzes und damit auch dessen Bedeutung für den hier betrachteten Kontext lässt sich mit den Ausführungen von Kaufmann und Rosewitz (1983, S. 32) treffend zusammenfassen: „Politik - oder genauer ,Policy Making“ - vollzieht sich als ein in charakteristische Phasen zu zerlegender Prozeß, an dem eine Vielzahl von Akteuren (und zwar typischerweise in unterschiedlichen Phasen verschiedene Akteure!) beteiligt sind. Diese Akteure haben unterschiedliche Interessen und Einflussmöglichkeiten, die sie aber unter charakteristischen institutionellen Restriktionen („Verfahren“) zur Geltung bringen müssen, so daß das Spiel der Kräfte zu einem charakteristischen Abschluß - typischerweise zu einer Entscheidung - gelangt. Die Theorie des politischen Prozesses geht also über das klassische Zweck-MittelDenken hinaus, das stets einen einheitlichen Akteur voraussetzt, und versucht, die Vielfalt von Einflüssen und Verknüpfungen zu berücksichtigen. Sie hält aber am Grundgedanken der Gerichtetheit der politischen Interaktion fest, d. h. sie unterstellt bestimmte politische Ziele oder Programme, die in einer bestimmten Phase des politischen Prozesses formuliert und sodann in einer späteren Phase - der Implementation - in die Wirklichkeit umgesetzt werden sollen.“

Gerade für die Betrachtung der Aufgabenerfüllung in Kommunen und der sie bestimmenden Komplexität an Rahmenbedingungen erlauben Phasenmodelle also darüber nachzudenken, wie kommunales Policy Making letzten Endes durch eine stärkere Integration von prozessbestimmenden Akteuren aus Kommunalpolitik, Kommunalverwaltung sowie Kommunalwirtschaft 
wirksam unterstützt bzw. verbessert werden kann. ${ }^{2}$ Ziel einer policy-bezogenen Betrachtung sollte es daher sein, mögliche Antworten auf Fragen dergestalt zu geben, wie z. B. eine bestimmte Policy im Bereich der öffentlichen Daseinsvorsorge auf die kommunalpolitische Agenda kommt, wie und unter welchen Bedingungen kommunalpolitische Programme und Entscheidungen diesbezüglich zustande kommen, welche Instrumente zur Steuerung einer Policy genutzt werden, welche Kräfte auf deren Entwicklung einwirken und welche Veränderungen aus der Umsetzung resultieren - zusammenfassend also darum, wie sich das Erreichen kommunalpolitischer Ziele oder die Erfüllung öffentlicher Aufgaben über ein engmaschiges Netz an unterschiedlichen Institutionen, hier insb. in Form eines Zusammenspiels von Kommunalpolitik, Kernverwaltung und öffentlichen Unternehmen, vollzieht (grundlegend Jann 1981, S. 12).

In Abgrenzung zu der beschriebenen Policy-Dimension bezeichnet „Politics“ den mitunter konfliktreichen und durch gegensätzliche Interessen geprägten Prozess der Ver- und Aushandlung von Konflikten, verschiedenen Zielen und Inhalten sowie der Macht- und Einflussverteilung. Zentrale Ausgangspunkte einer politics-bezogenen Analyse sind daher Macht, Konflikt, Konsens und Durchsetzung (Jann 1984, S. 38; Mayntz 2010, S. 46). Für den hier behandelten Kontext geht es also konkret darum, eine Ausgewogenheit der Interessenverhältnisse zwischen Entscheidungsträgern aus Kommunalpolitik, Kommunalverwaltung und öffentlichen Unternehmen anzustreben. Damit ist die besondere Herausforderung verbunden, zum einen den „Primat der Politik" nicht in Frage zu stellen, zum anderen aber das Know-how und die Kompetenz öffentlicher Unternehmen auf den einzelnen Stufen des Policy Cycle kooperativ zu nutzen. In der „Sprache“ von Governanceprinzipien bzw. Koordinationsmechanismen geht es also darum, eine Balance zwischen einer hierarchisch angelegten politischen Steuerung und der kooperativen Einbindung dieser Akteure dort zu erreichen, wo es mit Blick auf die Gewährleistung einer nachhaltigen Daseinsvorsorge als sinnvoll erscheint - ohne Prinzipal-Agenten- bzw. Auftraggeber-Auftragnehmer-Beziehungen umzukehren (Röber 2008, S. 63ff.).

„Polity“, als dritte Dimension eines mehrdeutigen Politikbegriffs, bezeichnet den formalen Aspekt von Politik. Gemeint sind Ordnungen, Normen und Institutionen, in deren Rahmen kommunalpolitische Prozesse ablaufen - mit anderen Worten, die durch Verfassungen, Regeln und Normen sowie durch institutionelle Arrangements bestimmten politisch-administrativen Strukturen in einer Kommune (Jann 1984, S. 38; Röber 2008, S. 62). „Polity“ beinhaltet z. B. die für die Steuerung und Kontrolle öffentlicher Unternehmen als relevant einzustufende Ausgestaltung von formellen und informellen Institutionen und Normen, wie Gemeinde- oder Haushaltsordnungen, untergesetzliche Empfehlungen, wie Kodizes oder Beteiligungsrichtlinien, entscheidungsrelevante Organisationseinheiten, Gremien und Organe, aber auch kulturelle Aspekte. „Polity“ bezieht sich damit auf Fragen und Anforderungen der Zusammensetzung, Befähigung und schlussendlich des Zusammenwirkens der verschiedenen Organisationseinheiten, Gremien, Organe, Institutionen und Normen, die kommunalpolitische Aushandlungsprozesse beeinflussen. Dies schließt zum einen politische Ausschüsse (z. B. Beteiligungsausschuss) oder Lenkungsgremien (z. B. im Rahmen städtischer Strategieprozesse) ein, zum anderen ist „Polity“ auch auf Fragen nach der geeigneten Organisation des Beteiligungsmanagements oder der Aus-

2 Sicherlich spielen in diesem Zusammenhang auch Fragen der Bürgerbeteiligung eine Rolle. Dieser Aspekt steht hier allerdings nicht im Fokus der Betrachtung. 
gestaltung und des Zusammenwirkens von Leitungs- und Aufsichtsorganen in öffentlichen Unternehmen zu beziehen, bei denen es um eine geeignete Qualifikation (und hiermit weitere Anforderungen an eine Mandatsbetreuung) sowie funktionierende Anreizstruktur geht (Röber 2008, S. 62ff.; Bogumil 2011, S. 543; Papenfuß/Aufenacker 2011, S. 25ff.).

Vor dem Hintergrund der aufgezeigten konzeptionellen Aspekte soll es auf Grundlage einiger explorativer Befunde im Folgenden darum gehen, welche Charakteristika die einzelnen Phasen der Problemwahrnehmung, des Agenda Setting und der Politikformulierung auf kommunaler Ebene aufweisen können, welche Rolle öffentliche Unternehmen in diesen Phasen spielen und wie diese Rolle zu bewerten ist.

\section{III. Öffentliche Unternehmen in den Phasen der Problemwahrnehmung, des Agenda Setting und der Politikformulierung}

Die kommunale Problembearbeitung und -verarbeitung setzt zuallererst die Wahrnehmung eines (sozialen) Problems und das Erkennen der Notwendigkeit eines Steuerungsbedarfs voraus. Es geht um die Fragen, welche Probleme in einer Kommune festzustellen, welche Entwicklungen zu erwarten und welche normativen Verbindungen zu berücksichtigen sind (u. a. Jann 1981, S. 31). Auf Ebene der kommunalen Problemwahrnehmung und des Agenda Setting geht es vornehmlich um Formen der politischen Planung. Im Verständnis von Luhmann (1971, S. 66ff.) sind im Rahmen dieses kommunalen Planungsprozesses Entscheidungsprämissen festzulegen, um die spätere Situation der Politikformulierung besser strukturieren zu können, ohne jedoch die tatsächliche Entscheidung vorweg zu nehmen. Im Vordergrund stehen daher die Eingrenzung von Entscheidungsproblemen und die Bestimmung von Bedingungen zu deren Lösung. Die Fixierung bestimmter Entscheidungsprämissen wird neben Planung im Wesentlichen durch Aspekte der Organisation, Entscheidungskompetenz, Kommunikationsweise sowie Persönlichkeit beeinflusst (ebd.).

$\mathrm{Ob}$ und wie Themen auf die politische Agenda in einer Kommune kommen, hängt $\mathrm{u}$. a. von verschiedenen Interessen und Rationalitäten der beteiligten Akteure, Handlungskapazitäten sowie der zu einem bestimmten Zeitpunkt objektiv wahrzunehmenden Konjunktur bzw. Relevanz bestimmter Themen ab. Aufgrund von begrenzten Problemwahrnehmungs- und auch Problembearbeitungskapazitäten ist eine hinreichende Filterung der potenziellen kommunalpolitischen Themen als notwendig zu erachten (Liu u. a. 2010; Jann/Wegrich 2007, S. 45ff.; grundlegend Scharpf 1973 a; 1973 c).

Vor diesem Hintergrund können neben Akteuren aus der Kommunalpolitik verschiedene Akteure aus Kommunalverwaltung und der Kommunalwirtschaft bereits bei der Politikwahrnehmung und dem Agenda Setting einen entscheidenden Beitrag leisten, wenn es darum geht, gesellschaftlich artikulierte Probleme im Rahmen der kommunalen Aufgabenerfüllung zu definieren und in den Fokus der politischen Problemwahrnehmung zu rücken (Gains 2003, S. 55ff.; Birkland 2007, 70 f.). Dabei spielen vor allem öffentliche Unternehmen eine wichtige Rolle, da sie durch ihr vielfach vorhandenes Know-how und ihre in den letzten Jahren zunehmend entwi- 
ckelte Beratungskompetenz mittlerweile sehr gut in der Lage sind, zu einer Abgrenzung relevanter Problemkomplexe beizutragen (Friedländer 2016, S. 106).

Öffentliche Unternehmen stehen durch die Bereitstellung öffentlicher Güter und Dienstleistungen in den einzelnen Politikfeldern, ähnlich wie Ämter der Kernverwaltung, in direktem Bürger- bzw. Kundenkontakt. Artikulierte Bedarfe, sei es durch ein hinreichendes Selbstverständnis und eine als notwendig erachtete Darstellung des Unternehmens selbst oder aber durch tatsächlich artikulierte Kundenbedürfnisse im Rahmen von Befragungen oder Veranstaltungen, können direkt durch die Unternehmensleitungen, durch Mitglieder der Aufsichtsgremien oder über den Austausch mit den jeweiligen Beteiligungsverwaltungen in den Fokus der politischen Problemwahrnehmung gebracht werden. Hierfür sei beispielhaft auf solche Formate, wie Stadtwirtschaftstage (z. B. Wissenschaftsstadt Darmstadt), Tage der offenen Tür oder aber Fahrgastbefragungen verwiesen, in deren Rahmen sich städtische Unternehmen mit ihren Leistungen den Bürgern vorstellen, die Möglichkeit zum gegenseitigen Informationsaustausch angeboten wird oder Kunden- bzw. Bürgerbedarfe abgefragt werden.

Solche Maßnahmen lassen sich, abgeleitet aus der konzeptionellen Literatur, als eine Form des Issue Raising beschreiben, wo es gezielt darum geht, die Fokussierung auf bestimmte Themen (z. B. die Relevanz der kommunalen Wirtschaft für Stadt und Region) bewusst zu erhöhen. Damit wird vor Augen geführt, dass die Stufe der Problemwahrnehmung im Verständnis einer Outside Initiation in der Regel ein in der Öffentlichkeit stattfindender Prozess ist (Jann/Wegrich 2007, S. 46).

Neben den genannten Beispielen bewegen sich öffentliche Unternehmen vielfach in sehr gut informierten Interessenvertretungen und Netzwerken, im Rahmen derer sie sich umfassend über innovative Ansätze und Branchenentwicklungen informieren und diese mit auf die politische Agenda bringen. Zu solchen gehören z. B. Verbände und von diesen organisierte Veranstaltungen (z. B. Kongresse des Verbands kommunaler Unternehmen) oder Kooperationsnetzwerke innerhalb von Kommunen, die dazu dienen können, lokale Rahmenbedingungen und Entwicklungsmöglichkeiten der Daseinsvorsorge im Rahmen des kommunalpolitischen Prozesses mitzugestalten (z. B. Kölner Netzwerk zur Daseinsvorsorge oder Mehrwert-Initiative Berlin). Wie der folgende Auszug aus den aktuellen Beteiligungsgrundsätzen der Stadt Wien sicherlich auch für viele andere Städte und Gemeinden exemplarisch unterstreicht, erhofft man sich von derartigen Netzwerkstrukturen positive Effekte und erachtet eine engere Zusammenarbeit zwischen Kommunalpolitik, -verwaltung und -wirtschaft als maßgebend für die Politikgestaltung und Leistungserbringung: „Die Stadt Wien bildet gemeinsam mit ihren vielfältigen Beteiligungsunternehmen das Haus Wien - ein kooperatives Netzwerk unter dem Dach der gemeinsamen Eigentümerin Stadt Wien. Dies schafft zusätzlichen Nutzen, der beispielsweise in Form von Forschungs- und Entwicklungskooperationen, einer Lehrlingsoffensive, der digitalen Agenda oder innovativen öffentlichen Beschaffungsstrategien in allen Teilbereichen der Stadt Wien sichtbar wird." (Stadt Wien 2016, S. 7). ${ }^{3}$

3 Hinsichtlich der Aussagekraft der Befunde aus der Dokumentenanalyse ist allerdings auf eine kleine Einschränkung hinzuweisen. Über die analysierten Dokumenteninhalte kann nicht immer zweifelsfrei auch auf deren tatsächliche Umsetzung geschlossen werden. Insofern ist nicht auszuschließen, dass es sich in einigen Fällen eher um ,,dokumentierte Lippenbekenntnisse“ als um tatsächlich umgesetzte Prozesse oder durchgeführte Maßnahmen handelt. 
Einige Führungskräfte in der kommunalen Kernverwaltung nehmen daher durchaus wahr, so ein Ergebnis der mündlichen Befragung, dass öffentliche Unternehmen neben Politik und Kernverwaltung mittlerweile eine entscheidende Rolle dabei spielen, bestimmte mit der öffentlichen Aufgabenerfüllung in Verbindung stehende Policies auf die politische Agenda zu bringen. Eine Einbeziehung öffentlicher Unternehmen in die Policy-Gestaltung wird gerade auch deshalb begrüßt, weil hierdurch Akzente aus der betrieblichen Wirklichkeit oder, wie oben skizziert, Erfahrungen aus anderen Kooperationen und Netzwerken aufgegriffen werden können. Um aktuelle Themen zu erörtern und den Austausch zwischen den Akteuren zu intensivieren, werden in einigen Fällen Treffen zwischen Politik, Verwaltungsspitze und Geschäftsführern bzw. Vorständen der öffentlichen Unternehmen durch die jeweilige Beteiligungsverwaltung initiiert. Die regelmäßig stattfindenden Treffen werden nach Rückmeldung gerade von den Unternehmensleitungen als gute Gelegenheit wahrgenommen, um sich über aktuell wichtige politische Prozesse und Themen innerhalb der Stadtverwaltung und des Stadtrates zu informieren und auszutauschen.

So veranstaltet bspw. die HEAG Holding AG der Wissenschaftsstadt Darmstadt ,,jährlich ein Stadtwirtschaftsforum, bei dem sich ausgewählte städtische Beteiligungen den politisch Verantwortlichen aus Stadtverordnetenversammlung und Magistrat vorstellen und ihre aktuellen Projekte präsentieren. Das Ziel dieser regelmäßigen Veranstaltung ist die Förderung des gegenseitigen Austauschs zwischen Politik und Stadtwirtschaft sowie die Erhöhung der Transparenz." (Wissenschaftsstadt Darmstadt 2015, S. 62). Ähnlich institutionalisierte Formate veranstaltet auch das Land Berlin. Hier findet seit 2017 jährlich ein parlamentarisches Treffen mit Vertretern der Landesunternehmen statt (sog. Mehrwert-Abende), in dessen Rahmen sich Unternehmen und Kooperationsinitiativen den Mitgliedern der Berliner Regierung vorstellen.

Allerdings ist in den Gesprächen einschränkend darauf hingewiesen worden, dass die Einbeziehung öffentlicher Unternehmen in der Phase der Problemwahrnehmung und des Agenda Settings nicht dazu genutzt werden darf, die Hierarchiestrukturen zwischen Gesellschafter und Unternehmen zu durchbrechen. D. h., bei einer Einflussnahme auf Policy-Prozesse durch öffentliche Unternehmen muss aus Sicht der Steuerungssubjekte zwischen der Einbindung von Knowhow auf der einen und der Durchsetzung von (zu) partikulären Interessen, Handlungsrationalitäten bzw. Impulsen auf der anderen Seite sauber unterschieden werden.

In der Phase der Politikformulierung spielen neben „klassischen“ hierarchischen Koordinationsformen (insb. Beschluss von kommunalpolitischen Zielen oder dem Haushalt) ebenfalls Verhandlungsprozesse und das kooperative Zusammenspiel zwischen Kommunalpolitik, Kommunalverwaltung und öffentlichen Unternehmen eine wichtige Rolle (Jann/Wegrich 2007, S. 49; Sidney 2007, S. 79ff.; für den Kontext von Bundesämtern und Bundesagenturen Bach 2012, S. $211 \mathrm{ff}$.). Es geht in diesem Zusammenhang vor allem um sich in Größe und Zeit unterscheidende Zusammenschlüsse von Akteuren, d. h. um Policy- oder Issue-Netze, in denen kollektive Entscheidungen herbeigeführt werden sollen (Mayntz 1993, S. 40 und 46).

In der Literatur wird darauf hingewiesen, dass die Phase der Politikformulierung für eine genauere empirische Analyse schwer zugänglich ist, da emergente Eigenschaften neuer Policies sehr komplex sein können. Politikformulierung sowie der Übergang zur Politikwahrnehmung sind von stärkeren Unübersichtlichkeiten geprägt, wodurch konkrete Aussagen über beteiligte Akteure und deren Interessen schwer möglich sind. Solche Situationen können in besonderem 
Maße zu mikropolitischem Taktieren und intransparentem Verhalten tendieren, da partikuläre Interessen in der Regel für den Einzelnen im Vordergrund stehen und er diese Interessen auch durchsetzen möchte. Hinzu kommt, dass Phasen der Politikformulierung sowohl von offener und verdeckter Machtausübung geprägt sein können („two faces of power“) als auch von einem Verhalten, mit dem versucht wird, einen bestimmten inhaltlichen Status quo zu sichern und weitere neue Politikformulierungen nach Möglichkeit zu verhindern (,nondecision-making“) (Bachrach/Baratz 1962, S. 947ff.; Crozier/Friedberg 1979; Brüggemeier/Felsch 1992).

Trotz dieser Schwierigkeiten ist davon auszugehen, dass eine breite Beteiligung von Akteuren wichtige Grundlage dafür sein kann, dass formulierte Kommunalziele später auch von den relevanten Akteuren getragen und tatsächlich umgesetzt werden. Denn kooperative Strategieprozesse können zumindest teilweise dazu beitragen, Know-how, Kompetenzen sowie unterschiedliche Interessen und Handlungslogiken der Akteure zu berücksichtigen, Widersprüche und Konflikte aufzuzeigen sowie Grenzen der Kooperationsbereitschaft erkennen zu lassen (Schedler/Siegel 2004, S. 80). Auch aus politikwissenschaftlicher Sicht erhofft man sich von einem kooperativen Policy-Prozess auf dieser Stufe einen wichtig Beitrag dafür, kommunalpolitische Entscheidungsstrukturen stärker unter Verhandlungsdruck zu setzen (Bogumil 2011, S. 544).

Vor diesem Hintergrund ist es für die Phase der Politikformulierung als besonders wichtig einzustufen, dass sich relevante Akteure aus Gemeindevertretung, Kommunalverwaltung und öffentlichen Unternehmen im Dialogprozess darüber einigen, welche Leitvorstellungen und Ziele in der Kommune erreicht werden sollen (z. B. Bremeier/Brinckmann/Killian 2006, S. 169). In der Terminologie der Stakeholder-Theorie geht es in diesem Kontext darum, dass unterschiedliche und sich teilweise widersprechende Ansprüche relevanter Stakeholder in der Stadt ausreichend berücksichtigt, die Beziehungen zwischen verschiedenen Stakeholder-Gruppen analysiert werden und auf die (sich ändernden) Ansprüche dieser Gruppen adäquat reagiert wird, um politische Durchführbarkeit und städtische Legitimität zu stärken (Friedländer 2016, S. 100 f.).

Damit kann begründet werden, warum Städte, die Strategieprozesse durchlaufen (haben), vielfach Dialog-, Strategie- bzw. Sachverständigenforen mit relevanten Stakeholder-Gruppen durchführen, um politische Themen im Rahmen der öffentlichen Aufgabenerfüllung tatsächlich auch als solche zu formulieren. Die Landeshauptstadt Potsdam hat im Rahmen des Leitbildprozesses z. B. bewusst darauf geachtet, sowohl Politik als auch Kommunalwirtschaft in den Strategieprozess einzubinden. Hierzu existiert eigens ein Lenkungsgremium: „Um auch die Verbindung zur Politik und den kommunalen Unternehmen sicherzustellen, wird das Lenkungsgremium, das für die Leitbildentwicklung initiiert wurde, weitergeführt. Somit sind die Fraktionsvorsitzenden und die Geschäftsführer der kommunalen Unternehmen in den Prozess der strategischen Steuerung aktiv eingebunden." (Landeshauptstadt Potsdam 2016, S. 3).

Im Idealfall kann eine kooperative Einbindung öffentlicher Unternehmen in die beschriebenen Policy-Phasen dazu führen, dass Ziele und Aufgaben dieser Unternehmen für einzelne Bereiche der kommunalen Daseinsvorsorge in städtischen Strategiedokumenten (verbindlich) festgelegt und definiert werden. So wird in dem sog. Arbeitsprogramm 2020 des Leipziger Oberbürgermeisters explizit auf den Beitrag öffentlicher Unternehmen zur kommunalen Zielerreichung Bezug genommen und Bürgern zumindest für die größten städtischen Unternehmen erläutert, welchen Beitrag diese in den einzelnen Bereichen der Daseinsvorsorge leisten (Stadt Leipzig 2014, S. 23ff.). Die darin enthaltenen Aussagen mögen auf den ersten Blick recht allgemein klingen. 
Analysiert man kommunale Strategiedokumente aber diesbezüglich genauer, stellt man fest, dass nur wenige Kommunen in derartigen Dokumenten überhaupt auf die Leistungserbringung ihrer Kommunalwirtschaft eingehen, geschweige denn dem Anspruch einer stärkeren (auch dokumentierten) Integration kommunaler Beteiligungen in städtische Zielsystematiken gerecht werden.

Im Kontext der Formulierung von Zielen und Strategien ist darüber hinaus die Stadtwirtschaftsstrategie 2020 der Wissenschaftsstadt Darmstadt und der HEAG Holding AG zu nennen. Dieses Strategiepapier ist im Jahr 2009 durch die Stadtverordnetenversammlung beschlossen worden und war das erste seiner Art in Hessen und eines der ersten in Deutschland. In dem Strategiedokument sind die städtischen Ziele für die unmittelbaren und mittelbaren Mehrheitsbeteiligungen Darmstadts formuliert. Sie sollen dazu beitragen, die wirtschaftliche Betätigung der Stadt ganzheitlicher zu betrachten. Daher wird auch denjenigen Unternehmen, an denen die Stadt zu weniger als $50 \%$ beteiligt ist, die Anwendung der Stadtwirtschaftsstrategie empfohlen (Ahrend/ Hoschek 2016, S. 130; Wissenschaftsstadt Darmstadt 2015, S. 1ff.). Ausgehend von einem städtischen Leitbild, welches von Akteuren aus Politik, Stadtwirtschaftskoordination sowie den Geschäftsleitungen der Unternehmen erarbeitet wurde, formuliert das Strategiepapier leistungsbezogene (z. B. Sicherstellung von Kernleistungen der Daseinsvorsorge für die Stadt) und unternehmensbezogene Ziele (z. B. Steigerung der Wertschöpfung) sowie Kernziele für einzelne Geschäftsfelder und Unternehmen des Stadtkonzerns (u. a. für das städtische Beteiligungsmanagement) (Wissenschaftsstadt Darmstadt 2015, S. 3ff.).

In der Freien und Hansestadt Bremen möchte man im Rahmen eines umfassenden Reformprozesses noch einen Schritt weitergehen und versuchen, öffentliche Unternehmen in die haushalterische Produkt- und Produktgruppensystematik zu integrieren. In dem Reformprogramm wird die „Aufnahme der öffentlichen Unternehmen, Eigengesellschaften, -betriebe und Sondervermögen in den Produktgruppenhaushalt" (Freie und Hansestadt Bremen 2017, S. 76) daher als ein explizites Ziel genannt. Sollte dieses Modernisierungsvorhaben tatsächlich umgesetzt werden, wäre damit ein wichtiger Baustein gesetzt, der vielfach geforderten integrierten Gesamtsteuerung öffentlicher Aufgaben auf kommunaler Ebene einen wesentlichen Schritt näher zu kommen (Friedländer 2019).

Blickt man nochmals auf die gesamte Phase der Politikformulierung, ist - so wurde es zumindest aus Perspektive ausgelagerter Aufgabenträger im Rahmen der mündlichen Befragung geäußert - für die kommunalpolitische Praxis davon auszugehen, dass die Einflussmöglichkeiten auf die konkrete Ausgestaltung einer kommunalen Policy durch öffentliche Unternehmen, wie auch „Dritte“, an der Stelle enden, wo die erarbeiteten Vorlagen den Geschäftsbereich des (Ober-)Bürgermeisters verlassen und an den Stadt- oder Gemeinderat weitergeleitet werden. Genau an dieser Stelle sind dann auch die o g. Unübersichtlichkeiten in der Phase der Politikformulierung auszumachen, die schlichtweg eine „Black Box“ im kommunalpolitischen Prozess darstellen. Für sich genommen bietet dieses Verfahren wenig Raum für Kritik. Es kann aber dazu führen, dass kommunalpolitische Strategiebeschlüsse am Ende nicht das beinhalten, was zu Beginn des politischen Prozesses die bezweckte Intention eines bestimmten Policyoder Issue-Netzes war (z. B. die kommunalpolitische Schwerpunktlegung auf einen bestimmten Bereich der öffentlichen Daseinsvorsorge). Aus Sicht der Kommunalpolitik kann dieses Vorgehen sicherlich so gedeutet werden, dass es einen gewissen Schutz vor einem unkontrollierbaren 
Issue Raising bietet. Die politischen Hauptakteure werden sich letztlich auch bei kooperativen Prozessen ihre Machtposition zur Orientierung, Vermittlungsorganisation und Letztentscheidung vorbehalten und die ihnen zur Verfügung stehenden Politics-Instrumente dementsprechend einsetzen. Dennoch kann diese Form der Prozessgestaltung bei externen Akteuren zu einer gewissen Resignation führen, speziell für öffentliche Unternehmen sogar zu einer Verstärkung dysfunktionaler Dezentralisierungs- und Autonomiebestrebungen. Vor diesem Hintergrund sollte für die Einbeziehung öffentlicher Unternehmen stets eine Ausgewogenheit zwischen der Einbringung sinnvoller Politikvorschläge und einem „handhabbaren“ Policy-Prozess gewahrt werden.

Gerade an dieser Schnittstelle, so wird im folgenden Abschnitt gezeigt, kann dem Beteiligungsmanagement eine wichtige Rolle zugesprochen werden, wenn es darum geht, Konflikte im kommunalpolitischen Prozess auszubalancieren und kooperativ ausgerichtete Entscheidungsprozesse zu befördern.

\section{Beteiligungsmanagement als Unterstützungsfunktion im kommunalpolitischen Prozess}

„Beteiligungsmanagement“ ist durch eine komplexe Aufgabenstruktur geprägt. Es beinhaltet Planungs-, Kontroll-, Koordinations-, Steuerungs- sowie Informations- und Beratungsaufgaben, um Kommunalvertretung und Verwaltungsführung bei der politischen Steuerung der Unternehmen zu unterstützen. Aufgrund der Sach- und Formalziellogik öffentlicher Unternehmen geht es bei der Steuerung dieser Unternehmen im ersten Schritt darum, dass die Gemeinde bzw. Stadt als Gesellschafter festlegen muss, welcher öffentliche Zweck mit einem Unternehmen mittel- bis langfristig verfolgt werden bzw. welchen Beitrag ein Unternehmen zu Erreichung kommunalpolitischer Ziele leisten soll. In dem dafür notwendigen Zielbildungs- bzw. Aushandlungsprozess kann das Beteiligungsmanagement als Bindeglied zwischen der politischen Steuerung durch Kommunalvertretung und Verwaltungsspitze auf der einen Seite und der operativen Leistungserbringung durch die Unternehmen auf der anderen Seite verstanden werden. Einem kommunalen Beteiligungsmanagement ist in diesem Zusammenhang eine wichtige Unterstützungsrolle zuzumessen, wenn es darum geht, Informationen für Abstimmungs- und Entscheidungsprozesse zwischen Stadtspitze und Unternehmen zu bündeln, aufzubereiten und zu kommunizieren, diese Prozesse zu organisieren und zu moderieren sowie Konfliktpotenziale zwischen den verschiedenen Akteuren auszutarieren (Huffmann 2011, S. 394ff.; KGSt 2012, S. 12ff.; Schaefer 2004, S. 227). Mit Rückgriff auf die verschiedenen Dimensionen des Politikbegriffs hat ein Beteiligungsmanagement im kommunalpolitischen Prozess daher eine besonders hohe Politics- wie Polity-Bedeutung.

Wie die Befragungsergebnisse unterstreichen, ist die Rolle des Beteiligungsmanagements in kommunalpolitischen Prozessen ein besonders gutes Beispiel dafür, dass Governanceprinzipien in der Regel in hybriden Formen existieren und die Grenzen zwischen Hierarchie, Netzwerk und Verhandlungssystem sowie Wettbewerb in den meisten Fällen verschwimmen (Friedländer 2019, S. 267ff.). Hervorzuheben ist aus Sicht von Akteuren aus der kommunalen Beteiligungs- 
verwaltung bspw. das Mit-, Neben- oder Untereinander von formellen Strukturen (z. B. Gesellschafteranordnung, Wirtschafts- und Mittelfristplanung, Gremienbeschluss) und informeller Koordination (z. B. regelmäßig stattfindende Jours fixes oder bilaterale Gespräche zwischen Beteiligungsverwaltung und Geschäftsführungen).

Nach den Interviewergebnissen scheint die Koordination über Vertrauen oder „Vier-AugenPrinzip" gerade in kleineren Kommunen eine funktionierende Form der Beteiligungssteuerung zu sein. In diesen Fällen können kommunale Strategieprozesse gemeinsam von den Hauptverwaltungsbeamten und Geschäftsführern der Beteiligungen initiiert und durchgeführt werden. Dadurch wissen die Geschäftsführer, was im Rathaus gewollt ist, umgekehrt besteht in der Kernverwaltung Kenntnis darüber, worin für die Unternehmen die derzeitigen Herausforderungen bestehen. Zur Aufrechterhaltung des Kommunikationsnetzes finden regelmäßige Jours fixes zwischen dem Bürgermeister (der in den meisten Gesellschaften gleichzeitig Aufsichtsratsvorsitzender ist) und Geschäftsführern oder Betriebsleitern statt. Hierdurch entsteht ein enges personelles Verflechtungssystem in den Gremien. Ziel ist es, ein Steuerungs- und Policy-Netz $\mathrm{zu}$ erzeugen, in dem insbesondere Bürgermeister und erster Stadtrat über alle Beteiligungsbereiche hinreichend gut informiert sind. Das enge Netz gewährleistet sowohl bei den Steuerungssubjekten als auch bei den -objekten, dass sämtliche Akteure über vertiefte Kenntnisse in den jeweiligen Bereichen verfügen, mitunter bis hin zu operativen Fragestellungen. In Hinblick auf Steuerungs- und Koordinationsprozesse ermöglichen diese Netzwerkstrukturen gut funktionierende „Kaskadeneffekte“, bei denen Problemwahrnehmung und -bearbeitung, selbst bei der Abwesenheit wichtiger Entscheidungsträger, sehr gut funktioniert.

Für die Formulierung kommunalpolitischer Ziele bedarf es umfassenderer Diskurse sowie einer gewissen analytischen Sach- und Prozessunterstützung. Die Entwicklung kommunaler Zielsysteme ist von vielen Politics-Einflüssen geprägt, wird von Widersprüchen begleitet und erfolgt selten konfliktfrei.

Daher ist im Rahmen der Befragung für das Thema der Integration kommunaler Beteiligungen in Ziel- und Steuerungssystematiken darauf hingewiesen worden, dass kommunale Zielsysteme zu keiner allzu starken Einschränkung des unternehmerischen Verständnisses und Handelns führen dürfen. Zwar ist unbestritten, dass öffentliche Unternehmen an konkrete kommunalpolitische Ziele und Programme zu binden sind. Dennoch sollte die selbständige Wirtschaftsführung dieser Unternehmen und damit auch die Autonomie ihrer Geschäftsführungen durch eine Gesamtstrategie nicht unverhältnismäßig eingeschränkt werden.

In dieser Forderung zeigt sich deutlich, dass jegliche kommunale Strategieorientierung die Aspekte der Steuerbarkeit und Steuerungsfähigkeit sowie der Steuerungsaktivität und des Steuerungsergebnisses von Anfang an berücksichtigen muss. Andernfalls, so wurde es von einem Befragungsteilnehmer verdeutlicht, besteht die Gefahr, dass die ohnehin langwierigen und mitunter konfliktreichen Strategieprozesse vielfach scheitern - nämlich dann, wenn sich die Geschäftsführungen öffentlicher Unternehmen von diesen Strategieprozessen abwenden, Ergebnisse nicht anerkennen und sich Dezentralisierungs- und Autonomiebestrebungen in der Folge verstärken.

Beteiligungsmanagement ist, so kann zusammengefasst werden, ein vergleichsweise stark von Verflechtungen geprägter Bereich; dies trifft umso mehr für dessen Rolle in kommunalen Policy-Prozessen zu. Informelle Wege des Informationsaustauschs, der Abstimmung und des Inter- 
essenausgleichs können bei (noch) überschaubaren Beteiligungsstrukturen innerhalb politischer Prozesse einer unkontrollierbaren Verselbstständigung öffentlicher Unternehmen oder einer Entscheidungsblockade durch ihre Geschäftsführungen entgegenwirken. Darüber hinaus wird mit den Schilderungen unterstrichen, dass kooperative und dialogorientierte Verfahren sicherlich auch einen gewissen Umfang an stabilisierenden Regeln benötigen, was für jeden einzelnen Akteur innerhalb der beschriebenen Strukturen vor allem die freiwillige Beschränkung der eigenen Handlungsfreiheiten und das Zugeständnis an divergierende Interessen zwischen Beteiligungssteuerung und Unternehmensleitung beinhaltet.

In größeren Kommunen sieht die Situation dagegen etwas anders aus. Zwar scheint die o g. Situationsbeschreibung auf den ersten Blick auch ein wünschenswerter Ansatz für die „Steuerung“ von Policy-Prozessen in größeren Kommunen. Allerdings sollte von einem naiven Glauben an die Übertragbarkeit informeller Mechanismen gerade mit Blick auf das mitunter sehr differenzierte und komplizierte Spannungsverhältnis zwischen Eigentum und Management im kommunalen Beteiligungsmanagement Abstand genommen werden.

Einer der Befragten wies in diesem Zusammenhang darauf hin, dass Einflussmöglichkeiten und -formen des Beteiligungsmanagements im Rahmen kommunalpolitischer Prozesse insbesondere im Zusammenhang mit der Größe des städtischen Konzerns zu betrachten und bewerten sind. Diese Feststellung ist aus konzeptioneller Perspektive nachvollziehbar. Kleinere Kommunen verfügen in den meisten Fällen sowohl über eine geringere Anzahl an Beteiligungen als auch über eine geringere Anzahl an Akteuren, die mit Fragen der Kommunalwirtschaft und ihrer Integration in politische Prozesse befasst sind. Somit können Abstimmungen direkter zwischen Verwaltungsspitze und Leitungsebene der Beteiligungen stattfinden. In diesem Klima kommen die Vorteile kooperativer und vertrauensbasierter Mechanismen am ehesten zur Entfaltung (u. a. höherer Grad an Flexibilität, Freiwilligkeit und Integration), wobei auch hier die möglichen Defizite weicherer Koordinationsformen (insbesondere Anfälligkeit für Missbrauch) berücksichtigt werden sollten. In der Umkehr ist eine formalisiertere und hierarchiegestützte Rolle des Beteiligungsmanagements innerhalb kommunalpolitischer Prozesse in größeren Kommunen durchaus mit den Vorteilen einer hierarchischen Organisation zu begründen. Hat man es mit großstädtischen Beteiligungsstrukturen zu tun, gewinnen Aspekte der Regelgebundenheit, Berechenbarkeit oder auch der arbeitsteiligen Aufgabenbewältigung zwischen den verschiedenen Steuerungssubjekten und Steuerungsobjekten im politischen Prozess an Bedeutung. So ist es, vereinfacht ausgedrückt, schwer vorstellbar, eine dreistellige Zahl an städtischen Beteiligungen rein über Vertrauen, ,Vier-Augen-Prinzip“ und informelle Gespräche in kommunale Zielbildungsprozesse einbinden zu wollen. In solch komplexen Strukturen kommt es umso mehr auf die Reduktion von Unsicherheiten und Transaktionskosten an. Hierarchisch-formale Strukturen können und sollten gerade bei der Einbindung sehr komplexer Organisationsgebilde in kommunale Policy-Prozesse ein stabilitätsförderndes Grundgerüst bieten, in dem sich die funktionalen Effekte der anderen Governanceformen - hier insbesondere der Kooperation und Verhandlung - bestmöglich entfalten können. 


\section{Fazit}

Eine phasengestützte Policy-Analyse ermöglicht es, den Beitrag öffentlicher Unternehmen zur Daseinsvorsorge auf kommunaler Ebene nicht nur allein auf die Leistungserbringung zu reduzieren, sondern Untersuchungen zusätzlich auf die vorgelagerten kommunalpolitischen Entscheidungsstufen auszudehnen. Mit Rückgriff auf die unterschiedlichen Kategorien des Politikbegriffs und ausgewählte Aspekte der Koordination öffentlicher Aufgaben, wird die Grundlage geschaffen, Akteurskonstellationen im Spannungsverhältnis zwischen Kommunalpolitik, Kommunalverwaltung und Kommunalwirtschaft zu betrachten und deren Zusammen- bzw. Entgegenwirken auf den einzelnen Stufen des kommunalpolitischen Prozesses besser verstehen und deuten zu können.

Die explorativen Befunde zeigen, dass öffentliche Unternehmen an kommunalpolitischen Prozessen beteiligt sind, sich in mehr oder weniger institutionellen Policy-Netzwerken bewegen und Themen der öffentlichen Daseinsvorsorge auf die kommunalpolitische Agenda bringen können. Öffentliche Unternehmen sind für die betrachteten Fälle daher durchaus als politische Akteure zu bezeichnen, die in einzelnen kommunalen Modernisierungsprozessen einen wichtigen Gestaltungsbeitrag leisten.

Die Einbindung öffentlicher Unternehmen in kooperative Aushandlungsprozesse wird von den befragten Entscheidungsträgern befürwortet, solange sie in erster Linie der Einbeziehung von Know-how und Kompetenzen dient und weniger der Durchsetzung partikulärer Interessen. Wenn es nämlich um Fragen nach dem „Wann“, „Wo“ und „Wie viel“ an Einbeziehung öffentlicher Unternehmen in politische Prozesse geht, werden politische Funktionsträger, als die maßgeblichen politischen Akteure, ihre Machtposition zur Letztentscheidung durchzusetzen wissen. Öffentliche Unternehmen bewegen sich daher gewisser Maßen im Schatten des politisch-administrativen Systems und im Spannungsverhältnis politischer Interessenlagen.

Dennoch, so zeigen es die Befunde, können kooperativere Strukturen in einzelnen Arenen kommunalpolitischer Modernisierungsprozesse einen Beitrag dazu leisten, begrenzten Problemwahrnehmungs- und Problembearbeitungskapazitäten entgegenzuwirken und die Integration ausgelagerter Aufgabenträger in kommunale Zielsystematiken zu fördern. Gelingt es, solche Modernisierungsinitiativen als verbindliche Reformprogramme zu formulieren, kann man dem Ziel einer kommunalen Gesamtsteuerung öffentlicher Aufgaben durchaus einen wichtigen Schritt näher kommen.

In diesem Zusammenhang ist dem Beteiligungsmanagement eine wichtige Unterstützungsrolle zuzumessen, wenn es darum geht, Koordinationsmechanismen in Abhängigkeit unterschiedlicher Organisations- und Akteurskonstellationen so zu wählen, dass Abstimmungs- und Entscheidungsprozesse zwischen Stadtspitze und Unternehmen moderiert sowie Konfliktpotenziale zwischen den verschiedenen Akteuren austariert werden. Beteiligungsmanagement kann daher als wichtiges Bindeglied zwischen der politischen Steuerung durch Kommunalvertretung und Verwaltungsspitze auf der einen Seite und der operativen Leistungserbringung durch die Unternehmen auf der anderen Seite verstanden werden. Es hat im kommunalpolitischen Prozess daher eine besondere Politics- wie Polity-Bedeutung. 
Für die akademische Diskussion bleibt mit Blick auf die einleitend aufgeworfenen Forschungslücken festzuhalten, die Rolle öffentlicher Unternehmen im kommunalen Institutionengeflecht und im politischen Prozess nicht mehr nur auf Begrifflichkeiten, wie „Dezentralisierung“, „Autonomie“ oder „Perforierung“ zu reduzieren. Vielmehr sollten die bestehenden Kooperationsstrukturen zwischen Kommunalpolitik, Kommunalverwaltung und Kommunalwirtschaft, deren Funktionsweisen sowie deren Beitrag für die Organisation und Erbringung öffentlicher Daseinsvorsorge in entsprechenden Untersuchungen zukünftig stärker Berücksichtigung finden.

\section{Abstract}

Benjamin Friedländer; State-Owned-Enterprises and their Control and Coordination in the Local Policy Process: Conceptualisation and Findings from an Interview Survey and a Document Analysis

State-owned enterprises; control and coordination of SOEs; policy; local policy process; polity; politics; governance mechanism

With regard to the scientific literature, it can be noted that the discussion on state-owned enterprises (SOEs) primarily focuses on management aspects as well as institutional economic analyses. There is a lack of classification into policy science and political framework conditions. This is particularly evident for the contribution of SOEs to local policy processes and for the actors involved in their control and coordination. Based on a conceptual framework from a policy, politics and polity perspective as well as explorative findings from interviews with local decision makers and a document analysis the questions addressed here are: What role do SOES play in the different phases of the policy process? How can role and interaction patterns be evaluated? What influence does the control and coordination of municipal holdings have on decision-making processes, conflict resolution, and consensus building?

\section{Literaturverzeichnis}

Ahrend, Klaus-Michael und Markus Hoschek (2016), Gemischtwirtschaftliche Unternehmen in der Stadtwirtschaft der Wissenschaftsstadt Darmstadt - ein Praxisbericht, in: Gemischtwirtschaftliche Unternehmen, Bestandsaufnahme und Perspektiven für Forschung und Praxis, hrsg. von Ulf Papenfuß und Christoph Reichard, Beiheft 48 der Zeitschrift für öffentliche und gemeinwirtschaftliche Unternehmen, S. 127-146.

Bach, Tobias (2012), The involvement of agencies in policy formulation: Explaining variation in policy autonomy of federal agencies in Germany, in: Policy \& Society, vol. 31, no. 3, pp. 211-222.

Bachrach, Peter und Morton S. Baratz (1962), Two Faces of Power, in: The American Political Science Review, vol. 56, no. 4, pp. 947-952.

Birkland, Thomas A. (2007), Agenda Setting in Public Policy, in: Handbook of Public Policy Analysis: Theory, Politics, and Methods, hrsg. von Frank Fischer, Gerald J. Miller und Mara S. Sidney, Boca Raton u. a., pp. 63-78.

Bogumil, Jörg (2011), Die Umgestaltung des Verhältnisses von Politik und Verwaltung, in: Handbuch zur Verwaltungsreform, 4. aktualisierte und ergänzte Auflage, hrsg. von Bernhard Blanke, Frank Nullmeier, Christoph Reichard und Göttrik Wewer, Wiesbaden, S. 536-545. 
Bremeier, Wolfram, Hans Brinckmann und Werner Killian (2006), Public Governance kommunaler Unternehmen: Vorschläge zur politischen Steuerung ausgegliederter Aufgaben auf der Grundlage einer empirischen Erhebung, Düsseldorf.

Brüggemeier, Martin und Anke Felsch (1992), Mikropolitik, in: Die Betriebswirtschaft, 52. Jg. Heft 1, S. 133-136.

Crozier, Michel und Friedberg, Erhard (1979), Macht und Organisation. Die Zwänge kollektiven Handelns, Königstein/Ts.

Freie und Hansestadt Bremen (2017), Programm zukunftsorientierte Verwaltung. Masterplan 2017/2018, Bremen.

Friedländer, Benjamin (2016), Integrierte Gesamtsteuerung von Kernverwaltung und öffentlichen Unternehmen, in: Nachhaltige und wirkungsorientierte Steuerung öffentlicher Unternehmen, hrsg. von Michèle Morner und Ulf Papenfuß, Speyer, S. 95-106.

Friedländer, Benjamin (2019), Kommunale Gesamtsteuerung öffentlicher Aufgaben: Bestandsaufnahme, Bewertung und Perspektiven, Diss., Wiesbaden.

Gains, Francesca (2003), Executive Agencies in Government: The Impact of Bureaucratic Networks on Policy Outcomes, in: Journal of Public Policy, vol. 23, no. 1, pp. 55-79.

Grossi, Giuseppe und Christoph Reichard (2008), Municipal Corporatization in Germany and Italy, in: Public Management Review, vol. 10, no. 5, pp. 597-617.

Heclo, Hugh (1972), Policy Analysis, in: British Journal of Political Science, vol. 2, no. 1, pp. 83-108.

Hesse, Mario, Thomas Lenk und Tim Starke (2017), Investitionen der öffentlichen Hand. Die Rolle der öffentlichen Fonds, Einrichtungen und Unternehmen, Gütersloh.

Huffmann, Harald (2011), Kommunales Beteiligungsmanagement, in: Handbuch der kommunalen Wissenschaften und Praxis, Band 2: Kommunale Wirtschaft, Dritte, völlig neu überarbeitete Auflage, hrsg. von Thomas Mann und Günter Püttner, S. 379-408, Wiesbaden.

Jann, Werner (1981), Kategorien der Policy-Forschung, Speyer.

Jann, Werner (1984), Verwaltung im politischen Prozeß, in: Verwaltungsrundschau, 2/84, S. 37-43.

Jann, Werner und Kai Wegrich (2007), Theories of the Policy Cycle, in: Handbook of Public Policy Analysis: Theory, Politics, and Methods, hrsg. von Frank Fischer, Gerald J. Miller und Mara S. Sidney, Boca Raton u. a., pp. 43-62.

Kaufmann, Franz-Xaver und Bernd Rosewitz (1983), Typisierung und Klassifikation politischer Maßnahmen, in: Implementation politischer Programme II, hrsg. von Renate Mayntz, Opladen, S. 25-49.

KGSt (1993), Das Neue Steuerungsmodell, Bericht Nr. 5/1993, Köln.

KGSt (2012), Steuerung kommunaler Beteiligungen, Bericht 3/2012, Köln.

Landeshauptstadt Potsdam (2016), Leitbild für die Landeshauptstadt Potsdam, Vorlage 16/SVV/0275, Potsdam.

Liu, Xinsheng, Eric Lindquist, Arnold Vedlitz und Kenneth Vincent (2010), Understanding Local Policymaking: Policy Elites' Perceptions of Local Agenda Setting and Alternative Policy Selection, in: The Policy Studies Journal, vol. 38 , no. 1, pp. 69-91.

Luhmann, Niklas (1971), Politische Planung. Aufsätze zur Soziologie von Politik und Verwaltung, Opladen.

Mayntz, Renate (1983), Zur Einleitung: Probleme der Theoriebildung in der Implementationsforschung, in: Implementation politischer Programme II, hrsg. von Renate Mayntz, S. 7-24, Opladen.

Mayntz, Renate (1993), Policy-Netzwerke und die Logik von Verhandlungssystemen, in: Policy-Analyse. Kritik und Neuorientierung, hrsg. von Adrienne Héritier, Sonderheft 24/1993 der Zeitschrift Politische Vierteljahresschrift, S. 39-56.

Mayntz, Renate (2010), Governance im modernen Staat, in: Governance - Regieren in komplexen Regelsystemen. Eine Einführung, 2., aktualisierte und veränderte Auflage, hrsg. von Arthur Benz und Nicolai Dose, Wiesbaden, S. 37-48.

Naschold, Frieder, Dietrich Budäus, Werner Jann, Erika Mezger, Maria Oppen, Arnold Picot, Christoph Reichard, Erich Schanze und Nikolaus Simon (2000), Leistungstiefe im öffentlichen Sektor. Erfahrungen, Konzepte, Methoden, 2. unveränderte Auflage, Berlin.

Papenfuß, Ulf und Marcel Aufenacker (2011), Organisationsmodelle und Personaleinsatz im öffentlichen Beteiligungsmanagement - Eine Analyse auf Grundlage von Experteninterviews in 75 deutschen Großstädten, in: Zeitschrift für öffentliche und gemeinwirtschaftliche Unternehmen, 34. Jg., Heft 1, S. 25-51.

Röber, Manfred (2008), Die Sphäre des Politischen - ein blinder Fleck in der Public Corporate Governance?, in: Public Corporate Governance: Bestandsaufnahme und Perspektiven, hrsg. von Christina Schaefer und Ludwig Theuvsen, Beiheft 36 der Zeitschrift für öffentliche und gemeinwirtschaftliche Unternehmen, S. 57-68.

Röber, Manfred (2012), Institutionelle Differenzierung und Integration im Kontext des Gewährleistungsmodells, in: Institutionelle Vielfalt und neue Unübersichtlichkeit. Zukunftsperspektiven effizienter Steuerung öffentlicher Aufgaben zwischen Public Management und Public Governance, hrsg. von Manfred Röber, Berlin, S. 15-27.

Schaefer, Christina (2004), Steuerung und Kontrolle von Investitionsprozessen. Theoretischer Ansatz und Konkretisierung für das öffentliche Beteiligungscontrolling, Wiesbaden.

Scharpf, Fritz W. (1973 a), Komplexität als Schranke der politischen Planung, in: Planung als politischer Prozeß. Aufsätze zur Theorie der planenden Demokratie, hrsg. von Fritz W. Scharpf, Frankfurt am Main, S. 73-113. 


\section{Öffentliche Unternehmen und Beteiligungsmanagement im kommunalpolitischen Prozess}

Scharpf, Fritz W. (1973 b), Verwaltungswissenschaft als Teil der Politikwissenschaft, in: Planung als politischer Prozeß. Aufsätze zur Theorie der planenden Demokratie, hrsg. von Fritz W. Scharpf, Frankfurt am Main, S. 9-32.

Scharpf, Fritz W. (1973 c), Planung als politischer Prozeß, in: Planung als politischer Prozeß. Aufsätze zur Theorie der planenden Demokratie, hrsg. von Fritz W. Scharpf, Frankfurt am Main, S. 33-72.

Scharpf, Fritz W. (2000), Interaktionsformen. Akteurszentrierter Institutionalismus in der Politikforschung. Opladen.

Schedler, K./Siegel, J.P. (2004): Strategisches Management in Kommunen, Düsseldorf.

Schröter, Eckhard und Manfred Röber (2017), Public Managers in Germany: Between Patronage and Professionalism, Paper presented at the Annual Conference of the International Research Society on Public Management to be held from April 19-21, 2017 at the Corvinus University, Budapest.

Sidney, Mara S. (2007), Policy Formulation. Design and Tools, in: Handbook of Public Policy Analysis: Theory, Politics, and Methods, hrsg. von Frank Fischer, Gerald J. Miller und Mara S. Sidney, Boca Raton u. a., pp. S. 79-87.

Stadt Leipzig (2014), Leipzig! Arbeitsprogramm 2020, Leipzig.

Stadt Wien (2016), Beteiligungsgrundsätze, Wien.

Wissenschaftsstadt Darmstadt (2015), Stadtwirtschaftsstrategie 2020 der Wissenschaftsstadt Darmstadt. Geschäftsfeldübergreifende Ziele und Erwartungen an die Geschäftsfelder und die Unternehmen, Darmstadt. 